\title{
Synthesis and processing of improved graphite-molybdenum-titanium composites by colloidal route and spark plasma sintering
}

\author{
M. Suárez ${ }^{\text {a, }}$, D. Fernández-González ${ }^{\text {a, }}$,* L.A. Díaz ${ }^{\text {a }}$, A. Borrell ${ }^{\text {b }}$, J.S. Moya ${ }^{\text {a }}$, A. Fernández ${ }^{\text {a }}$

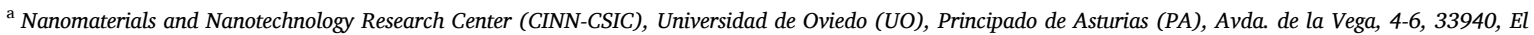 \\ Entrego, Spain \\ ${ }^{\mathrm{b}}$ Instituto de Tecnología de Materiales, Universitat Politècnica de València (UPV), Camino de Vera S/N, 46022, Valencia, Spain
}

\section{A R T I C L E I N F O}

\section{Keywords:}

Spark plasma sintering

Composites

Carbon

Carbides

\begin{abstract}
A B S T R A C T
Graphite-molybdenum-titanium composites might attract significant interest as heat sinks for different sector applications due to their excellent thermal, electrical and mechanical properties. This type of composites was already studied but following the powder metallurgy and spark plasma sintering (SPS) route. The aim of the present investigation is to study a new route of fabrication of these composites to improve their properties: colloidal synthesis and SPS process. The results obtained for graphite- 10 vol\% Mo- 1 vol\% Ti composites prepared by both routes are compared. It has been proved that the properties of the composites are significantly improved by the colloidal route compared with the powder metallurgy route, i. e. electrical conductivity by a factor of 3 , thermal properties by a factor of 8 and bending strength by a factor of 4 .
\end{abstract}

\section{Introduction}

Graphite-molybdenum composites have been subject of research for many years due to the excellent thermal-mechanical properties conferred by the graphite and molybdenum carbides, which are formed when sintered together, combined with a low density $\left(\sim 2.5 \mathrm{~g} / \mathrm{cm}^{3}\right)$, refractory character, high thermal stability and good thermal and electrical conductivities. Graphite itself exhibits good thermal and electrical conductivities, which explains some of its potential applications in electrodes, batteries, heat sinks, etc. However, it is an anisotropic material, and when it is pressed, planes of atoms in the basal plane tend to orientate in the direction perpendicular to the pressing, which gives better thermal-mechanical properties in this direction. For its part, molybdenum, when finely dispersed in the graphite matrix, can improve mechanical resistance of graphite as inhibits basal planes slip and fracture of the composite [1]. Moreover, molybdenum carbides exhibit metal-like structure [2] and, in this context, electrical and thermal conductivities do not get worse when the graphite-molybdenum composite is produced.

The addition of reactive metals allows strengthening the carbons and, specially, the graphite. In the case of graphite and molybdenum, several researchers have investigated the addition of molybdenum (carbides) to strengthen graphite. White and Pontelandolfo appreciated an improvement of thermal, electrical, and mechanical properties of graphite-molybdenum carbide composites to be used for hightemperature nuclear and aerospace applications prepared at temperatures that involved liquid phase [3-5]. They prepared by conventional molding the graphite-carbide bodies that were subsequently fired at $1800{ }^{\circ} \mathrm{C}$. Finally, the composites were prepared by hot working, which involves the free compressional deformation of a fabricated body unrestrained by a die (there is a free surface from which the excess of liquid can be squeezed). Harada and Rubin [6] investigated different mixtures of graphite-metals and graphite-metal carbides, including molybdenum, for which they studied the temperature when carbides appear and analyzed their electrical and mechanical properties. They prepared the composites by hot pressing of binary mixtures of carbon (petroleum coke was used as graphite source) and metal or metal carbide. Powder mixtures were dry blended for $16 \mathrm{~h}$, and they were later hot pressed at temperatures of around $2000{ }^{\circ} \mathrm{C}$. Matthews and Jenkins studied the interaction of molybdenum and graphite at high temperature $\left(2000-3000{ }^{\circ} \mathrm{C}\right)[7]$. This work provided useful details about the molybdenum diffusion in carbon. Graphite-molybdenum composites are used for extreme conditions applications (rocket nozzles [8]; collimators [9] or particle accelerators [10] that are used in the Large Hadron

\footnotetext{
* Corresponding author.

$* *$ Corresponding author.

E-mail addresses: m.suarez@cinn.es (M. Suárez), d.fernandez@cinn.es (D. Fernández-González).
} 
Collider at CERN [11]) due to the extraordinary thermal and electrical properties conferred by the molybdenum added to the graphite. In this context, Mariani [9] studied molybdenum-copper-diamond, molybdenum carbide-graphite and molybdenum carbide-graphite-carbon fibers composites. Molybdenum-graphite composites were prepared by rapid hot pressing $(10 \mathrm{~min})$ at temperatures of $1700^{\circ} \mathrm{C}$ with constant applied pressure of $45 \mathrm{MPa}$ under vacuum atmosphere $\left(10^{-4} \mathrm{mbar}\right)$ with reducing atmosphere $\left(97 \% \mathrm{~N}_{2}-3 \% \mathrm{H}_{2}\right)$. Bertarelli and Bizarro [10] also studied the sintering at high temperature of molybdenum-ceramic composites (graphite, carbon fibers, silicon, silicon carbide or tungsten). They proposed mixing the components for about $2 \mathrm{~h}$, green compaction (10-20 MPa) and hot pressing (2500-2600 ${ }^{\circ} \mathrm{C}$, at maximum pressures of 35-45 MPa). Guardia-Valenzuela and collaborators [11] studied the spark plasma sintering of powders of graphite-molybdenum-titanium using commercial reagents. Powder mixture was prepared in a 3D mixing machine, dry and without any mixing media, and then were compacted into a green body at up to 300 $\mathrm{MPa}$ in a uniaxial hydraulic press, inside a steel mould. Green compact was then sintered in the spark plasma sintering machine. Even when the main interest of graphite-molybdenum composites is related with the thermoelectrical properties with low density and good mechanical resistance, this type of composites has also found application in electrochemical processes [12]. Huang and colleagues synthesized graphite-molybdenum carbide nanocomposites by solid-state reaction with melamine and $\mathrm{MoO}_{3}$ as precursors under inert atmosphere. Pressed pellets of precursors were put in a horizontal alumina tubular furnace and flow of $\mathrm{N}_{2}$ and pellets were kept at $1673 \mathrm{~K}$ for $3 \mathrm{~h}$.

As opposed to the above-mentioned research articles, which are focused on graphite-molybdenum composites to be used in extreme conditions applications, the aim of the present work is to study the influence of using a colloidal route instead of the powder metallurgy one in the final properties of graphite-molybdenum-titanium composites after sintering them by spark plasma sintering.

\section{Processing and characterization of the nanocomposites}

\subsection{Synthesis of powders}

Graphite/molybdenum/titanium nanocomposites, containing 10 vol $\%$ Mo and 1 vol\% Ti, were prepared following two different synthesis routes:

(1) Colloidal route: Graphite powder (grade 93004, Asbury Carbons Company), consisting of crystalline natural graphite with spheroidal-flake morphology with an average particle size in volume of $d_{50}=6.007 \mu \mathrm{m}$ and a BET (Brunauer, Emmett and Teller) specific surface area of $16.84 \mathrm{~m}^{2} / \mathrm{g}$, was dispersed in absolute ethanol. A solution of molybdenum (V) chloride (SigmaAldrich, Spain) and a solution of titanium isopropoxide (ABCR, Spain) were added dropwise into the graphite dispersion. The slurry was first heated under magnetic stirring at $70{ }^{\circ} \mathrm{C}$ and subsequently in air at $120^{\circ} \mathrm{C}$ for $24 \mathrm{~h}$ to start the nucleation of molybdenum and titanium and eliminate any traces of alcohol. Dried powder was grounded and sieved through $<180 \mu \mathrm{m}$. A thermal treatment at $450{ }^{\circ} \mathrm{C}$ for $2 \mathrm{~h}$ in air was made in order to eliminate chloride traces.

(2) Powder mixing by attrition milling: Graphite from Asbury Carbons Company, molybdenum (purity of 99.5\%) from H. C. Starck, spherical particles with a mean particle size $\left(\mathrm{d}_{50}\right)$ of $5.5 \mu \mathrm{m}$ (mode: $3.5-5.5 \mu \mathrm{m}$ ) and titanium titanium (purity of 99.5\%) from Abcr GMbh, powder with irregular morphology and a particle size $<45 \mu \mathrm{m}$ ) were mixed in propanol liquid medium using an attrition mill for $1 \mathrm{~h}$. Later, the slurry was first heated under magnetic stirring at $70{ }^{\circ} \mathrm{C}$ and subsequently in air at $120^{\circ} \mathrm{C}$ for $24 \mathrm{~h}$ to eliminate any traces of alcohol. Dried powder was grounded and sieved through $<180 \mu \mathrm{m}$.

\subsection{Spark plasma sintering (SPS)}

Spark plasma sintering was chosen as sintering technique. Cylindrical samples with $40 \mathrm{~mm}$ were uniaxially pressed in a metallic mould at $15 \mathrm{MPa}$. Samples were inserted in the graphite mould and sintered at a heating rate of $25{ }^{\circ} \mathrm{C} \cdot \mathrm{min}^{-1}$ up to $1500{ }^{\circ} \mathrm{C}$ and $10^{\circ} \mathrm{C} \cdot \mathrm{min}^{-1}$ to the final temperature in a spark plasma sintering apparatus (FCT-HP D25/1) under an applied pressure of $30 \mathrm{MPa}$ during all sintering process and in a vacuum condition $\left(10^{-1} \mathrm{mbar}\right)$. The final temperature achieved was $2000{ }^{\circ} \mathrm{C}$ and holding time of $15 \mathrm{~min}$.

\subsection{Characterization techniques}

High-resolution transmission electron microscopy (HRTEM) was employed using a JEOL JEM $2100 \mathrm{~F}$ with an acceleration voltage of 200 $\mathrm{kV}$ with a field emission gun. This technique was used to check that molybdenum and titanium are chemically adhered to the surface of the graphite particles. Doped graphite by colloidal route was prepared for HRTEM by dispersing the final powder in ethanol. One drop of the dispersion was then dropped on the carbon-copper grid.

Powder and sintered samples were characterized by X-ray diffraction (XRD) on a Philips X' Pert Pro X-ray diffractometer by using $\mathrm{Cu}-\mathrm{K} \alpha$ radiation $(\lambda=0.15406 \mathrm{~nm})$ in the range from $5^{\circ}$ to $70^{\circ}$. The step size was $0.03^{\circ}$ and the step time $0.5 \mathrm{~s}$. The identification of the crystalline phases was made by using diffraction pattern files provided by JCPDS (International Centre for Diffraction Data).

The microstructural characterization of graphite/molybdenum/titanium nanocomposites synthetized by colloidal route and attrition milling was performed by field emission scanning electron microscopy (FESEM) on a Quanta FEG 650 in the backscattered electron mode. The grain size distribution is calculated form FESEM images using ImageJ software (ImageJ, U.S. National Institutes of Health, Bethesda, Maryland, USA).

Relative density of the sintered samples was determined by geometric model using Eq. (1).

$\rho(\%)=d / d_{t h} \cdot 100$

where $d$ is the measured-apparent density and $d_{t h}$ is the theoretical density obtained by helium pycnometer using AccuPyc 1330 V2.04 N.

Young's modulus has been estimated from measurements of the resonance frequency (Grindosonic MK7, Belgium). The flexural strength values of the tested samples were obtained from three-point bending tests with $3 \mathrm{~mm} \times 4 \mathrm{~mm} \times 40 \mathrm{~mm}$ polished surface bars using a universal mechanical testing machine (Shimadzu-series AGS-IX, Japan) with a $10 \mathrm{kN}$ load cell at a crosshead speed of $0.5 \mathrm{~mm} \mathrm{~min}{ }^{-1}$. Those properties were measured on perpendicular and parallel face of the samples. The flexure strength $(\sigma)$ was evaluated using equation (2). Three samples $(n=3)$ were used, and data were presented as mean \pm standard deviation (SD).

$\sigma=3 F L / 2 b d^{2}$

where $\mathrm{F}$ is the load (force) at the fracture point, $\mathrm{L}$ is the length of the support spam, b and d are the width and the thickness of the sample, respectively.

Electrical conductivity was determined on the sample by four-point probe measurement, using the equipment PSM1735 - NumetriQ Newtons 4th on parallel and perpendicular face to the uniaxial pressure. The average value of 10 measurements was considered as representative value of electrical conductivity of the sample.

The thermal diffusivity was measured according to the flash method using commercial equipment (LFA 457 MicroFlash, Netzsch). Samples with $10 \mathrm{~mm} \times 10 \mathrm{~mm} \times 3 \mathrm{~mm}$ dimensions were prepared and the thermal diffusivity was measured in the parallel face to the uniaxial pressure. Specific heat was determined to calculate the thermal 
conductivity using a C80 (Setaram Instrumentation) calorimeter equipped with stainless steel cells (S60/1413). Specific heat determination is carried out in continuous mode, using a heating ramp of $0.1{ }^{\circ} \mathrm{C} \cdot \mathrm{min}^{-1}$ from 20 to $40{ }^{\circ} \mathrm{C}$, with $2 \mathrm{~h}$ of stabilization at the start and end temperatures. The data processing was carried out using the Calisto Software. Thermal conductivity values in the perpendicular face to the uniaxial pressure were calculated using the electrical conductivity following equation (3) corresponding to Wiedemann-Franz law.

$k / \sigma=L T$

where $\mathrm{k}$ is the thermal conductivity, $\sigma$ is the electrical conductivity, $\mathrm{L}$ is the Lorenz number and $\mathrm{T}$ is the temperature.

\section{Results}

\subsection{Phase identification}

Fig. 1 shows the XRD pattern of samples of doped graphite powders by colloidal route and sintered by SPS. Graphite and $\mathrm{MoO}_{3}$ are detected when the powders are heated in air at $450{ }^{\circ} \mathrm{C}$ for $2 \mathrm{~h}$. After sintering in SPS at $2000{ }^{\circ} \mathrm{C}$, graphite and molybdenum carbide, MoC, are the mineralogical phases present in the samples prepared by colloidal route and powder mixing. MoC presents a cubic structure $(\mathrm{a}=4.27 \AA)$ and graphite peaks corresponding to very high levels of graphitization, matching the positions close to an ideal graphite structure $(\mathrm{a}=2.46400$ $\AA$ and $\mathrm{c}=6.71100 \AA$ ) [13]. Carbon donates electrons to metal atoms changing the coordination of metal atoms to conform the carbide structures. The metal-carbon bond is essentially of a metallic nature and contributes to stability of the carbide structure. At low temperatures Mo and graphite react to form hexagonal $\mathrm{Mo}_{2} \mathrm{C}$ and this reaction continues by the diffusion of carbon inside $\mathrm{Mo}_{2} \mathrm{C}$ to obtain MoC.

\subsection{Microstructural characterization}

High-resolution transmission electron microscopy (HRTEM) images at different magnification were taken to further investigate the configuration of reduced graphite- 10 vol\% Mo- 1 vol\% Ti synthesized by colloidal route at $850{ }^{\circ} \mathrm{C}$ in $\mathrm{H}_{2} / \mathrm{Ar}$ atmosphere where $\mathrm{Mo}_{2} \mathrm{C}$ nanoparticles are formed due to the atomic diffusion of carbon atoms inside

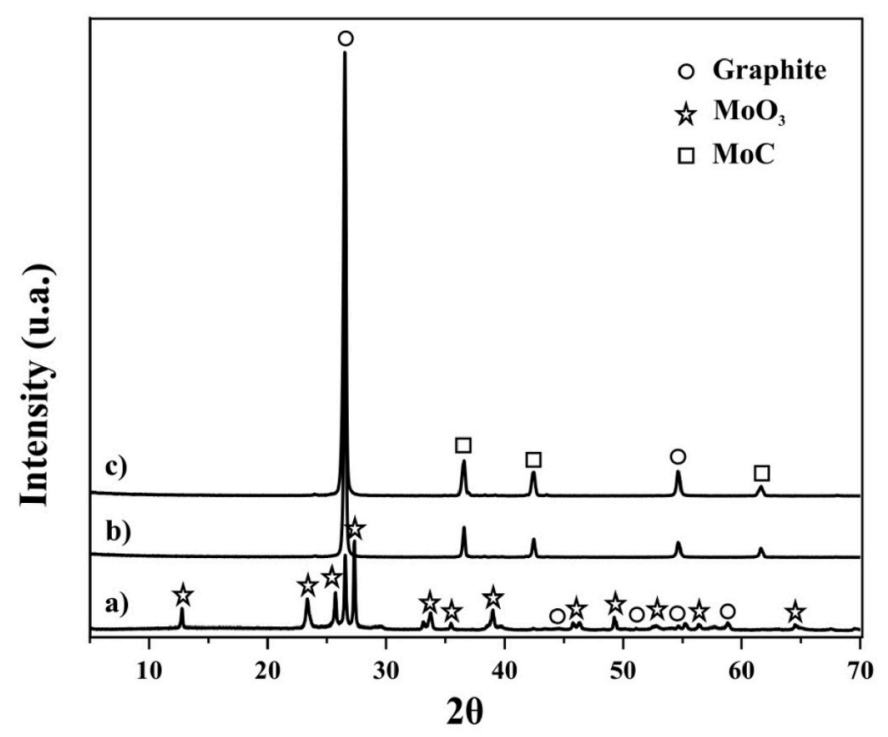

Fig. 1. X-ray diffraction patterns of doped graphite powder obtained by colloidal route and calcined at $450{ }^{\circ} \mathrm{C}$ for $2 \mathrm{~h}$ (a), doped graphite powder obtained by colloidal route and sintered by SPS (b) and doped graphite powder obtained by attrition milling and sintered by SPS (c). the molybdenum bcc lattice interstitials. Fig. 2a shows globularly shaped molybdenum carbide nanoparticles homogeneously dispersed and without hard agglomerates attached to the graphite layers, with sizes ranging from $3 \mathrm{~nm}$ to $50 \mathrm{~nm}$. Fig. $2 \mathrm{~b}$ shows a high-resolution micrograph of a molybdenum carbide crystalline particle of $20 \mathrm{~nm}$ where lattice fringes are clearly observed, indicate that the nanoparticles are structurally uniform, showing d-spacing of $0.224 \mathrm{~nm}$, representing the (101) crystalline plane of the $\mathrm{Mo}_{2} \mathrm{C}$.

Fig. 3 shows FESEM micrographs of polished doped graphite samples prepared by both attrition milling (Fig. $3 \mathrm{a}$ and b) and colloidal processing route (Fig. $3 \mathrm{c}$ and $\mathrm{d}$ ) and sintered by SPS. Both samples show a homogeneous distribution of second phase (white phase, molybdenum carbide) on the graphite matrix (black phase). The main difference between both samples is related to the particle size, $6 \mu \mathrm{m}$ in case of the sample prepared by attrition milling and $4 \mu \mathrm{m}$ for sample obtained by colloidal route and the distribution size. Fig. 4 shows the histogram representation of both samples obtained after a count of 65 particles. It shows that sample prepared by colloidal route presents a narrower granulometric distribution where the large fraction of particles is concentrated in the range $0-4$ microns. In case of attrition-milled sample, although a large fraction of particles in the $0-2$ microns' range is observed, it also contains a large proportion of them in the 4-10 microns range, due to the presence of MoC agglomerates. By ImageJ software it was possible to calculate the number of particles present in the area $\left(1.92 \mathrm{~mm}^{2}\right.$ ) observed in the FESEM micrographs (Fig. 3 a and c), turning out to be $1.2 * 10^{4}$ particles and $1.9 * 10^{4}$ particles for sample obtained by attrition milling and colloidal processing, respectively by the same percentage of dopant agent. Doping process has a great influence on the microstructure since the particle size is decreased and is reflected on the final properties of the sintered samples.

\subsection{Density}

Natural graphite powders compact into high density samples with very little applied pressures. This behaviour is caused by its selflubricating properties that allow intimate packing. However, when metal powders are cold compacted together with the graphite, the presence of these metallic particles reduces the graphite lubricating properties and therefore the packing of the composite.

Green density of the sample has been measured for both processing routes (attrition milling and colloidal route). Results are collected in Table 1. Higher green density value has been obtained in case of the samples sintered by colloidal route. Deformation temperature of the composite depends on the amount of disperse constituent. In case of samples processed by attrition milling, MoC nanoparticles are more agglomerated (they are less homogeneously distributed in the graphite matrix) increasing the particle size and the distance between the particles. A homogeneous distribution of the $\mathrm{MoC}$ in the graphite matrix for the sample obtained by colloidal route allows obtaining higher density values of the sintered samples.

\subsection{Mechanical characterization}

Strength and elastic properties have been evaluated and the obtained results are presented in Table 2 . The properties of molybdenum-doped graphite show a clear anisotropic behaviour since the properties depend on the orientation of the graphite flakes due to the stronger interatomic bond in the perpendicular direction to the applied pressure. The presence of MoC nanoparticles and the strong carbide-graphite bond in samples obtained by colloidal route allows obtaining better mechanical properties: in the bending strength test, samples improve up to $65 \%$ and $80 \%$ when the orientation is perpendicular and parallel, respectively to attrition milling. 

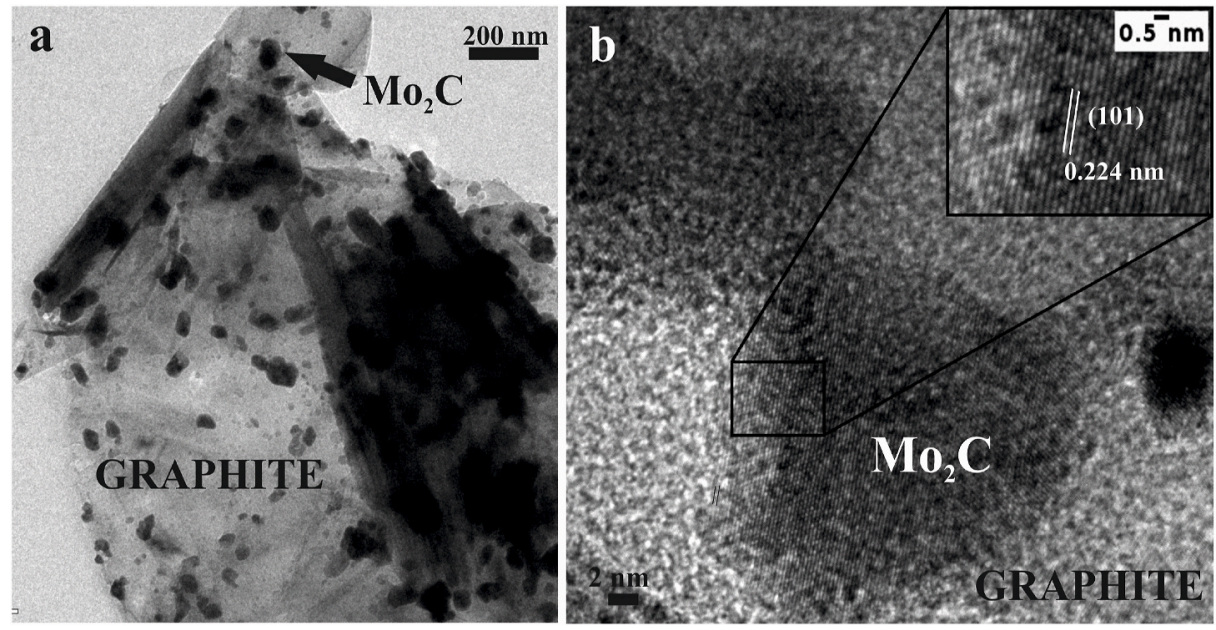

Fig. 2. HRTEM images of reduced modified graphite at $850{ }^{\circ} \mathrm{C}$ for $2 \mathrm{~h}$ and synthetized by colloidal route (a) and interplanar distance measured (b).
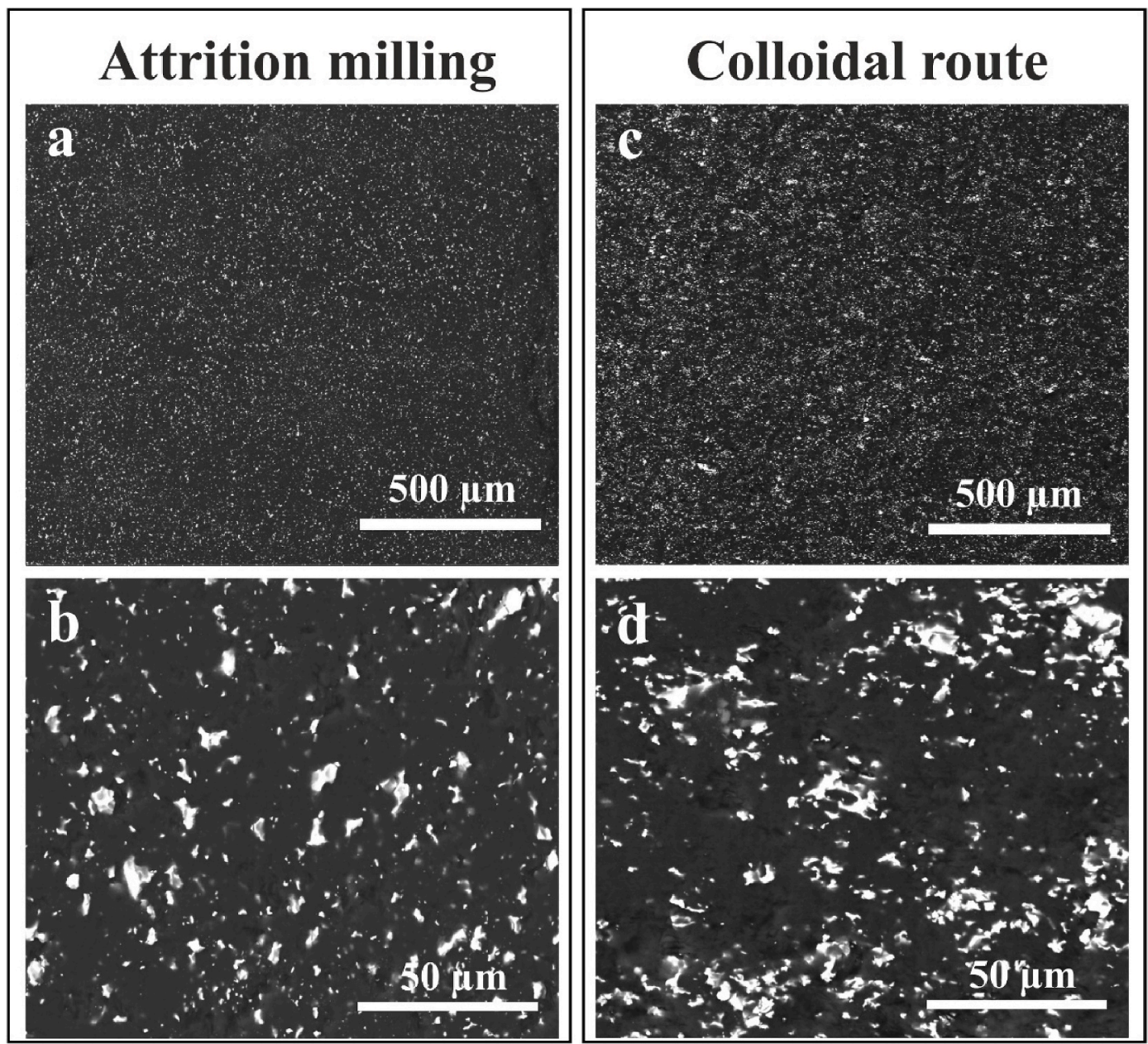

Fig. 3. FESEM polished surface of doped graphite by attrition milling (a) and colloidal route (b) and sintered by SPS.

\subsection{Electrical conductivity}

Superficial electrical conductivity measurements on sintered samples obtained by attrition milling and colloidal route are shown in Table 3. The electrical conductivity in the perpendicular direction to the applied pressure is higher than in the parallel direction in both types of samples.

\subsection{Thermal conductivity}

Thermal conductivity in graphite is dominated by phonon conduction and the values obtained follow the trend of the electrical conductivity being this value higher in the perpendicular direction to the pressure in both samples. Diffusivity in the perpendicular direction to the applied pressure cannot be determined by means of the equipment 

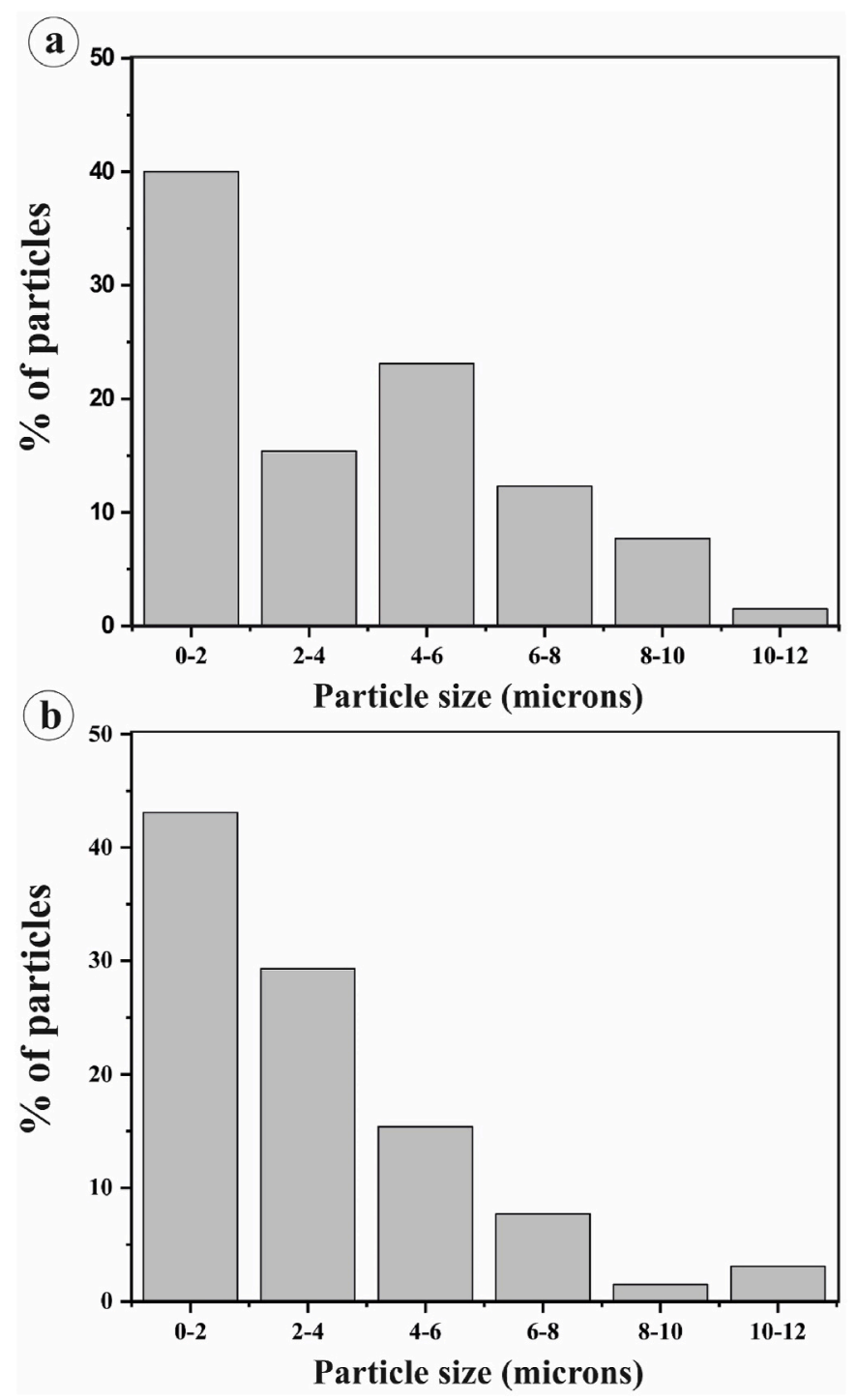

Fig. 4. Histogram of particle size distribution for graphite-Mo-Ti prepared by attrition milling (a) and colloidal route (b).

Table 1

Density of SPS sintered samples.

\begin{tabular}{lll}
\hline Reference & Green density $\left(\mathrm{g} / \mathrm{cm}^{3}\right)$ & SPS sample density (\%) \\
\hline Attrition milling & 1.54 & 92.1 \\
Colloidal route & 1.59 & 95.2 \\
\hline
\end{tabular}

Table 2

Mechanical properties of doped graphite samples obtained by attrition milling and colloidal route after sintered by SPS.

\begin{tabular}{llllll}
\hline \multirow{2}{*}{ Direction } & \multicolumn{2}{l}{ Attrition milling } & & & \multicolumn{2}{l}{ Colloidal route } \\
\cline { 2 - 3 } & $\perp$ & & & & \multicolumn{1}{l}{$\|$} \\
\hline Bending strength (MPa) & 48.09 & 29.90 & & 135.54 & 129.68 \\
Elastic modulus (GPa) & 79.57 & 54.25 & & 134.68 & 60.48 \\
\hline
\end{tabular}

model LFA 457 MicroFlash, Netzsch because of the thickness of the samples. Therefore, we use the Wiedemann-Franz law to indirectly determine the thermal conductivity by means of the electrical conductivity in this direction. The Lorenz Number is calculated by the thermal and electrical conductivities obtained in the perpendicular direction for
Table 3

Electrical properties of doped graphite samples obtained by attrition milling and colloidal route after sintered by SPS.

\begin{tabular}{|c|c|c|c|c|}
\hline \multirow[t]{2}{*}{ Direction } & \multicolumn{2}{|c|}{ Attrition milling } & \multicolumn{2}{|c|}{ Colloidal route } \\
\hline & $\perp$ & $\|$ & $\perp$ & $\|$ \\
\hline Electrical conductivity $\left(\mathrm{MS} \cdot \mathrm{m}^{-1}\right.$ ) & 0.32 & 0.26 & 0.99 & 0.16 \\
\hline
\end{tabular}

each sample, and it is assumed that the Lorenz Number is the same in both directions.

Results are collected in Table 4. As in the case of other properties, better results are obtained in the case of samples synthesized by colloidal route.

\section{Discussion}

Graphite reinforced with metallic materials is attracting significant interest for applications in the thermal domain (heat sinks for electronic devices or for high speed trains) due to the excellent properties. In the case of the graphite-molybdenum, there is no significant research, and most of it is mainly focused on applications for extreme conditions as different parts for rockets [5] or, most recently, for collimators to be used in the Large Hadron Collider at CERN [8]. However, graphite-molybdenum composites might find application in more common fields as those above indicated.

A main relevant conclusion is that the sintered material exhibits directional properties, with better properties in the direction perpendicular to the applied pressure. Furthermore discs sintered from powders obtained by the colloidal route have better properties than those sintered from powders obtained by simple mixing of powders, i. e. bending strength increases by a factor of 4 . The first question can be explained because graphite particles orient themselves perpendicular to the pressing direction by SPS, where the best properties are exhibited. Additionally, molybdenum carbides when finely dispersed as nanoparticles (see Fig. 2) inhibit basal planes slip through a pinning effect according to scheme shown in Fig. 5 and, therefore, enhance mechanical properties. The second question can be explained by the more homogeneous distribution of the molybdenum in the graphite matrix. This way, the improvement conferred by the molybdenum carbides is not impaired. And the third and significant question is that the sintering does not involve liquid phase, as in other research work with graphitemolybdenum composites, since temperature did not exceed $2000{ }^{\circ} \mathrm{C}$.

The dependence of the properties on the direction was observed by $\mathrm{J}$. L White and J. M. Pontelandolfo [2] in 1966. There is a strong interatomic bond in the perpendicular direction to the applied pressure of the molybdenum-doped graphite composite. Properties also depend on the doping method followed for preparing the materials. According to the results shown in Table 2, the bending strength, and the elastic modulus in the perpendicular direction to the applied pressure (136 MPa and 135 $\mathrm{GPa}$, respectively) are three and two times greater, respectively, in samples obtained by colloidal route than by attrition milling. These values are significantly greater if compared with those found in the literature for this kind of materials [8]. Three reasons would explain the increase of the strength: 1) increasing amounts of metal additions, 2) increasing pressing temperature and 3) decreasing carbide particle size.

Samples prepared by colloidal route have in perpendicular direction

Table 4

Thermal properties of SPSed doped graphite samples obtained by attrition milling and colloidal route.

\begin{tabular}{|c|c|c|c|c|}
\hline \multirow[t]{2}{*}{ Direction } & \multicolumn{2}{|c|}{$\begin{array}{l}\text { Attrition milling }+ \\
\text { SPS }\end{array}$} & \multicolumn{2}{|c|}{$\begin{array}{l}\text { Colloidal route }+ \\
\text { SPS }\end{array}$} \\
\hline & $\perp$ & $\|$ & $\perp$ & $\|$ \\
\hline Thermal conductivity $\left(\mathrm{W} \cdot \mathrm{m}^{-1} \cdot \mathrm{K}^{-1}\right)$ & 17.54 & 14.25 & 136.68 & 22.09 \\
\hline
\end{tabular}




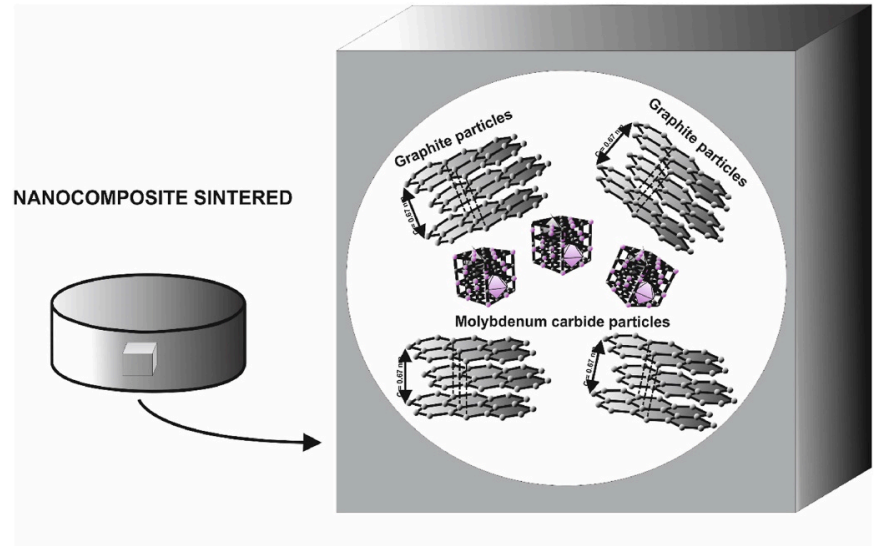

SECTION

Fig. 5. Distribution of molybdenum carbide on the graphite matrix in SPS samples.

an electrical conductivity about three times higher than samples obtained by attrition milling. This value is like that of sintered molybdenum-graphite composites prepared at temperatures as high as $2600{ }^{\circ} \mathrm{C}$ [8]. Colloidal route technique allows obtaining composites with high electrical conductivity at a temperature as low as $2000{ }^{\circ} \mathrm{C}$ (versus the $2600{ }^{\circ} \mathrm{C}$ usually used) since the good contact and homogeneous distribution between graphite carbon layers and MoC nanoparticles enhances the electrical conductivity of the composites in one direction [14].

\section{Conclusions}

Graphite-molybdenum-titanium composites were fabricated by spark plasma sintering from powders prepared by either colloidal route or powder mixing by attrition milling. Specimens were characterized by different techniques and mechanical, thermal and electrical properties are significantly enhanced (electrical conductivity by a factor of 3 , thermal properties by a factor of 8 and bending strength by a factor of 4) in the case of the composites prepared by means of the colloidal synthesis. Therefore, graphite-molybdenum-titanium composites could be prepared by the colloidal route and spark plasma sintering at temperature $<2000{ }^{\circ} \mathrm{C}$ in solid state, which is $600{ }^{\circ} \mathrm{C}$ lower than in other researches, with adequate properties for heat sink in different sector applications.

\section{Declaration of competing interest}

The authors declare that they have no known competing financial interests or personal relationships that could have appeared to influence the work reported in this paper.

\section{Acknowledgements}

This research was funded by the Spanish Ministry of Science and Innovation. Call Programa Estatal de $\mathrm{I}+\mathrm{D}+\mathrm{i}$ Orientada a los Retos de la Sociedad [RTI2018-102269-B-I00]. This research was also supported by a Juan de la Cierva Formación grant from the Spanish Ministry of Science and Innovation (MCINN) to Daniel Fernández-González [FJC2019041139-I]. A. Borrell acknowledges for her RYC-2016-20915. Authors are grateful to Ainhoa Macías San Miguel from Nanomaterials and Nanotechnology Research Center (CINN) for providing technical assistance.

\section{References}

[1] R.B. Matthews, D.B. Fischbach, G.M. Jenkins, Compaction and hot working of natural graphite-molybdenum carbide composite, in: Proceedings of the Third S.C. I. Carbon Conference, London, 1970, pp. 515-519.

[2] E. Parthe, V. Sadagopan, The structure of dimolybdenum carbide by neutron diffraction technique, Acta Crystallogr. 16 (3) (1963) 202-205.

[3] J. White, J. Pontelandolfo, Graphite-carbide materials prepared by hot-working with the carbide phase in the liquid state, Nature 209 (1966) 1018-1019.

[4] J. White, J. Pontelandolfo, Graphite-carbide materials prepared by hot-working with a dispersed liquid-carbide phase, Carbon 4 (1966) 305-314.

[5] J. White, J. Pontelandolfo, HWLC graphites prepared by hot-working extruded stock, Carbon 6 (1968) 1-2.

[6] Y. Harada, G.A. Rubin, Fabrication and characterization of metal carbide-graphite composites, in: Presented at the 68th Annual Meeting of the American Ceramic Society, May 9, 1966, Washington, D. C, 1966.

[7] R.B. Matthews, G.M. Jenkins, The high temperature interaction between molybdenum and graphite, J. Mater. Sci. 10 (1975) 1976-1990.

[8] R. Matthews, Deformation and Strengthening in Molybdenum Carbide - Natural Graphite Composite Materials, Ph.D. thesis, University of Wales, 1970.

[9] N. Mariani, Development of Novel, Advanced Molybdenum-Based Composites for High Energy Physics Applications, Ph. D. Thesis, Supervisor: Alessandro Bertarelli, Milan Polytechnic, Milan, Italy, 2014.

[10] A. Bertarelli, S. Bizzaro, Inventors; CERN - European Organization for nuclear research, Brevetti Bizz S.R.L., assignees. A molybdenum carbide/carbon composite and manufacturing method, Int. Pat. Appl. (2013). PCT/EP2013/072818 (2016).

[11] J. Guardia-Valenzuela, A. Bertarelli, F. Carra, N. Mariani, S. Bizzaro, R. Arenal Development and properties of high thermal conductivity molybdenum carbide graphite composites, Carbon 135 (2018) 72-84.

[[12]] K. Huang, K.C. Bi, C.S. Liang, S. Lin, W.J. Wang, T.Z. Yang, J. Liu, R. Zhang, D. Y. Fan, Y.G. Wang, M. Lei, Graphite carbon-supported $\mathrm{Mo}_{2} \mathrm{C}$ nanocomposites by a single-step solid state reaction for electrochemical oxygen reduction, Plos One 18 (2015) 1-11.

[[13]] J.Y. Howe, C.J. Rawn, L.E. Jones, H. Ow, Improved crystallographic data for graphite, Powder Diffr. 18 (2003) 150-154.

[[14]] K. Huang, K.C. Bi, C.S. Liang, S. Lin, W.J. Wang, T.Z. Yang, J. Liu, R. Zhang, D. Y. Fan, Y.G. Wang, M. Lei, Graphite carbon-supported $\mathrm{Mo}_{2} \mathrm{C}$ nanocomposites by a single-step solid state reaction for electrochemical oxygen reduction, PloS One 18 (2015) 1-11. 